TRANSACTIONS OF THE

AMERICAN MATHEMATICAL SOCIETY

Volume 363, Number 12, December 2011, Pages 6291-6308

S 0002-9947(2011)05223-2

Article electronically published on July 11, 2011

\title{
ON THE SIMPLY CONNECTEDNESS OF NONNEGATIVELY CURVED KÄHLER MANIFOLDS AND APPLICATIONS
}

\author{
ALBERT CHAU AND LUEN-FAI TAM
}

\begin{abstract}
We study complete noncompact long-time solutions $(M, g(t))$ to the Kähler-Ricci flow with uniformly bounded nonnegative holomorphic bisectional curvature. We will show that when the Ricci curvature is positive and uniformly pinched, i.e. $R_{i \bar{\jmath}} \geq c R g_{i \bar{\jmath}}$ at $(p, t)$ for all $t$ for some $c>0$, then there always exists a local gradient Kähler-Ricci soliton limit around $p$ after possibly rescaling $g(t)$ along some sequence $t_{i} \rightarrow \infty$. We will show as an immediate corollary that the injectivity radius of $g(t)$ along $t_{i}$ is uniformly bounded from below along $t_{i}$, and thus $M$ must in fact be simply connected. Additional results concerning the uniformization of $M$ and fixed points of the holomorphic isometry group will also be established. We will then consider removing the condition of positive Ricci curvature for $(M, g(t))$. Combining our results with Cao's splitting for Kähler-Ricci flow (2004) and techniques of $\mathrm{Ni}$ and Tam (2003), we show that when the positive eigenvalues of the Ricci curvature are uniformly pinched at some point $p \in M$, then $M$ has a special holomorphic fiber bundle structure. We will treat as special cases, complete Kähler manifolds with nonnegative holomorphic bisectional curvature and average quadratic curvature decay as well as the case of steady gradient KählerRicci solitons.
\end{abstract}

\section{INTRODUCTION}

In this paper we study the class of complete noncompact Kähler manifolds $\left(M^{n}, g\right)$ of complex dimension $n$ with bounded and nonnegative holomorphic bisectional curvature. Let $R$ be the scalar curvature and let

$$
k(r, x)=\frac{1}{V_{x}(r)} \int_{B_{x}(r)} R
$$

be the average of the scalar curvature over the geodesic ball with radius $r$ and center at $x$. In [11, [12] and [13] the authors obtained, among other things, the following uniformization results:

(1) If $k(r, x) \leq C /\left(1+r^{2}\right)$ for some $C$ for all $r$ and $x$, then the universal cover of $M$ is biholomorphic to $\mathbb{C}^{n}$.

Received by the editors October 12, 2009.

2010 Mathematics Subject Classification. Primary 53C44; Secondary 53C55, 58J37.

The first author's research was partially supported by NSERC grant no. \#327637-06.

The second author's research was partially supported by Earmarked Grant of Hong Kong \#CUHK403108.

(C)2011 American Mathematical Society Reverts to public domain 28 years from publication 
(2) Under a weaker assumption that $k(r, x) \leq C /(1+r)$ for some $C$ for all $r$ and $x$, the universal cover of $M$ is homeomorphic to $\mathbb{R}^{2 n}$ and is biholomorphic to a pseudoconvex domain in $\mathbb{C}^{n}$.

The results support the following conjecture due to Yau [33]: If $(M, g)$ has positive holomorphic bisectional curvature, then $M$ is biholomorphic to $\mathbb{C}^{n}$.

In this paper we want to discuss the structure of $M$ itself. In light of the above results, a reasonable approach is as follows. First one studies more closely the structure of the universal cover $\tilde{M}$. Then one would like to study properties of the first fundamental group, and hopefully these together will provide information about the structure of $M$. As in the previous works, we will study the Kähler-Ricci flow equation on $(M, g)$ :

$$
\frac{\partial g_{i \bar{\jmath}}}{\partial t}=-R_{i \bar{\jmath}} .
$$

By the works [26 and 28] of Shi, the Kähler-Ricci flow has a long-time solution $g(t)$ with uniformly bounded curvature under assumption (2) (and hence (1)) above. By the splitting result of Cao [7, $(\tilde{M}, \widetilde{g}(t))$, the pullback of $g(t)$ to the universal cover $\tilde{M}$, splits for all $t>0$ as $\tilde{M}=\mathbb{C}^{k} \times \tilde{N}$ and $\widetilde{g}(t)=g_{e} \times h(t)$, where $g_{e}$ is the standard metric on $\mathbb{C}^{k}$ and $h(t)$ has nonnegative holomorphic bisectional curvature and positive Ricci curvature. Hence we will begin our studies with longtime solutions $(M, g(t))$ to Kähler-Ricci flow, where $g(t)$ has uniformly bounded nonnegative holomorphic bisectional curvature and positive Ricci curvature. We will prove that:

If at some point $p \in M$, the eigenvalues of $\operatorname{Ric}(p, t)$ are uniformly pinched (i.e. the smallest eigenvalue of $\operatorname{Ric}(p, t)$ is at least $C$ times the largest eigenvalue for some $C>0$ independent of $t$ ), then $M$ is simply connected and is in fact biholomorphic to a pseudoconvex domain in $\mathbb{C}^{n}$.

See Theorems 2.1, 2.2 and 2.3. A particular case occurs when $(M, g)$ has average quadratic curvature decay and positive Ricci curvature in which case it is shown in Corollary 2.1 that $M$ is biholomrophic to $\mathbb{C}^{n}$. This generalizes previous results in [12] where the same result, in particular Corollary 2.1, is proved assuming in addition either that (1.2) has an eternal solution (i.e. $g(t)$ exists for $-\infty<t<\infty$ ) or that $t R$ is uniformly bounded in space-time. Note that either condition will imply that eigenvalues of $\operatorname{Ric}(p, t)$ are uniformly pinched; see [12] for example.

More generally, if we only assume the positive eigenvalues of $\operatorname{Ric}(p, t)$ are uniformly pinched, then using the above result on simple connectedness, we prove that:

$M$ is a holomorphic and Riemannian fiber bundle over $\mathbb{C}^{k} / \Gamma$, where $\Gamma$ is a discrete subgroup of the holomorphic isometry group of $\mathbb{C}^{k}$, with fiber either $\mathbb{C}^{n-k}$ or a pseudoconvex domain in $\mathbb{C}^{n-k}$.

See Theorem 3.1. In particular, the fiber is $\mathbb{C}^{n-k}$ when $(M, g)$ has average quadratic curvature decay, which we show in Corollary 3.1. Similar results under different conditions have been obtained in [25] and [30].

Our results are related to the second part of Yau's conjecture in [33]: If the holomorphic bisectional curvature is only nonnegative, then $M$ is biholomorphic to a complex vector bundle over a compact Hermitian symmetric space. In fact, we prove that if $n-k=1$ in the above situation, then $M$ is actually a line bundle over $\mathbb{C}^{k} / \Gamma$. In order to prove this, we have to study more carefully the isometry group of a complete noncompact Kähler manifold $(M, g)$ with nonnegative holomorphic 
bisectional curvature and positive Ricci curvature. We prove that if there is a long-time solution $g(t)$ to Kähler-Ricci flow with uniformly bounded nonnegative holomorphic bisectional curvature and positive uniformly pinched Ricci curvature, then any finite set of isometries of $M$ has a fixed point. Under the stronger assumption that (1.2) has an eternal solution or $t R(p, t)$ is uniformly bounded for all time, then one can prove the stronger result that there is a fixed point for all isometries of $(M, g)$. See Theorems 2.4 and 2.5. One might want to compare this with a theorem of Cartan which says that a compact subgroup of isometries of a Cartan-Hadamard manifold has a fixed point.

A special case of the above occurs when $g(t)$ is actually a gradient Kähler-Ricci soliton. Namely, there is a real-valued function $f$ with $f_{i j}=0$ such that $R_{i \bar{\jmath}}=f_{i \bar{\jmath}}$ (steady type) or after rescaling $-R_{i \bar{\jmath}}+g_{i \bar{\jmath}}=f_{i \bar{\jmath}}$ (expanding type). In the case of expanding type, it is known that $M$ is biholomorphic to $\mathbb{C}^{n}$ by [10]. For the case of steady type, under an additional assumption that the scalar curvature attains its maximum at some point, we prove that in most cases, $M$ is a holomorphic vector bundle or $\mathbb{C}^{k} / \Gamma$. More precisely, we prove that this is the case if the scalar curvature $R$ is maximal at $p \in M$ say, and the positive eigenvalues $\lambda_{1}, \ldots, \lambda_{l}$ of Ric at $p$ satisfy the Diophantine condition

$$
\sum_{i=1}^{l} m_{i} \lambda_{i} \neq \lambda_{j}
$$

for all $j$ and for all nonnegative integers $m_{i}$ such that $\sum_{i=1}^{l} m_{i} \geq 2$. See Theorem 4.1. The proof relies on the result of Bryant [2] on the existence of Poincaré coordinates. This is related to a result of Yang [32]: Suppose $M$ is a noncompact Kähler-Ricci soliton with nonnegative Ricci curvature such that its scalar curvature attains a positive maximum at a compact complex submanifold $K$ with codimension 1 and the Ricci curvature is positive away from $K$. Then $M$ is a holomorphic line bundle over $K$.

We now describe the organization of the paper. In $\S 2$ we study long-time solutions to Kähler-Ricci flow with bounded nonnegative holomorphic bisectional curvature and positive Ricci curvature. We will prove the existence of gradient KählerRicci soliton limits and consequences of this concerning the injectivity radius along the flow and the simple connectedness of $M$. We will also prove results on the existence of a fixed point of isometries. In $\S 3$ will prove the fiber bundle structure and line bundle structure of $M$. Finally, in $\S 4$ we prove the vector bundle structure on gradient Kähler-Ricci solitons. We will also prove a result on the volume growth of expanding gradient Ricci solitons in this section, which is related to the work of $\mathrm{Ni}[24,1]$

\section{LONG-TIME SOLUTIONS AND LOCAL LIMITS}

In this section we study limits of long-time solutions of the Kähler-Ricci flow (2.1) on complete noncompact Kähler manifolds with uniformly bounded nonnegative holomorphic bisectional curvature and positive Ricci curvature. We will investigate when such a solution subconverges, after rescaling, to a gradient Kähler-Ricci soliton limit, and we will derive a number of consequences from this. In Corollary 2.1 we treat the particular case of a complete noncompact Kähler manifold having

\footnotetext{
${ }^{1}$ This result (Proposition 4.1) is proved independently in 3 .
} 
bounded nonnegative holomorphic bisectional curvature and positive Ricci curvature which decays quadratically in the average sense. Our study of the limiting behavior of Kähler-Ricci flow in this section can be viewed as an extension of that in [11] and [12]. We begin with the following.

BASic Assumption 1: Let $M^{n}$ be a noncompact complex manifold of complex dimension $n$. Let $g(t)$ be a complete solution of the Kähler-Ricci flow

$$
\frac{\partial g_{i \bar{\jmath}}}{\partial t}=-R_{i \bar{\jmath}}
$$

on $M \times[0, \infty)$ with uniformly bounded nonnegative holomorphic bisectional curvature in space-time and with positive Ricci curvature.

Let $R(x, t)$ be the scalar curvature of $g(t)$ at $x$. Let $p \in M$ be a fixed point. Then we have the following possibilities: (1) $R(p, t) \geq c$ for some constant $c>0$ for all $t$; or $(2) \inf _{t \in[0, \infty)} R(p, t)=0$. In the second case, since $t R(p, t)$ is nondecreasing in time by a Li-Yau-Hamilton (LYH) type differential inequality of Cao 4, we have two subcases: (2a) $t R(p, t) \leq c$ for some constant $c>0$; and $(2 \mathrm{~b}) \lim _{t \rightarrow \infty} t R(p, t)=$ $\infty$. We address these cases separately in Theorems 2.1, 2.2 and 2.3 below.

First, we have the following lemma.

Lemma 2.1. Let $(M, g(t))$ be as in the BAsic Assumption 1. Let $p \in M$ be a fixed point. There exists $C>0$, such that for any $\frac{1}{4} \leq \alpha_{0} \leq \alpha_{1} \leq \frac{1}{2}$, any $\rho>0$, and any $T>0$ we have

$$
\frac{R\left(x, 4 \alpha_{1} T\right)}{R(y, 3 T)} \leq C \exp \left(\frac{\rho^{2}}{4}\right)
$$

for $x, y \in B_{4 \alpha_{0} T}(p, \rho \sqrt{T})$.

Proof. For $t>0$, fixed, define

$$
\hat{g}(s)=\frac{e^{s}}{t} g\left(t\left(1-e^{-s}\right)\right), 0 \leq s<\infty .
$$

Then we have

$$
\frac{\partial \hat{g}}{\partial s}=-\widehat{\operatorname{Ric}}+\hat{g}
$$

on $M \times[0, \infty)$. By the (LYH) type differential inequality in [4], for $x, y \in M$ and $0<s_{1}<s_{2}<\infty$ we have

$$
\frac{\widehat{R}\left(x, s_{1}\right)}{\widehat{R}\left(y, s_{2}\right)} \leq \frac{e^{s_{2}}-1}{e^{s_{1}}-1} \exp \left(\frac{1}{4} \Delta\right),
$$

where

$$
\Delta=\Delta\left(x, y ; s_{1}, s_{2}\right)=\inf _{\gamma} \int_{s_{1}}^{s_{2}}\left|\gamma^{\prime}(s)\right|_{\hat{g}(s)}^{2} d s
$$

and $\gamma$ is any curve from $x$ to $y$. Here $\widehat{R}$ is the scalar curvature and $\widehat{\text { Ric }}$ is the Ricci curvature of $\hat{g}$.

Let $\alpha_{1}=1-e^{-s_{1}}$ and $\alpha_{2}=1-e^{-s_{2}}$. Then $\widehat{R}\left(s_{i}\right)=\left(1-\alpha_{i}\right) R\left(\alpha_{i} t\right)$ for $i=1,2$, and we have $0<\alpha_{1}<\alpha_{2}$. Let $\alpha_{0}>0$ be fixed. Then $\hat{g}(s) \leq \frac{e^{s}}{t} g\left(\alpha_{0} t\right)$ for $\alpha_{0} \leq 1-e^{-s}$ because $g(t)$ is decreasing in $t$. Now for $x, y \in B_{\alpha_{0} t}(p, \rho \sqrt{t})$, let $\gamma$ be a minimal geodesic from $x$ to $y$ with respect to $g\left(\alpha_{0} t\right)$. If $\frac{1}{4} \leq \alpha_{0} \leq \alpha_{1} \leq \frac{1}{2}$ and $\alpha_{2}=\frac{3}{4}$, then

$$
\Delta\left(x, y ; s_{1}, s_{2}\right) \leq \frac{d^{2}}{t\left(s_{2}-s_{1}\right)^{2}} \int_{s_{1}}^{s_{2}} e^{s} d s \leq C_{1} \rho^{2}
$$


for some constant $C_{1}$ independent of $x, y, t, \rho, \alpha_{0}, \alpha_{1}$, where $d$ is the distance between $x, y$ with respect to $g\left(\alpha_{0} t\right)$. Hence

$$
\frac{R\left(x, \alpha_{1} t\right)}{R\left(y, \frac{3}{4} t\right)} \leq C_{2} \exp \left(\frac{\rho^{2}}{4}\right)
$$

for $x, y \in B_{\alpha_{0} t}(p, \rho \sqrt{t})$ and $\frac{1}{4} \leq \alpha_{0} \leq \alpha_{1} \leq \frac{1}{2}$. Here $C_{2}$ is independent of $x, y, t, \rho, \alpha_{0}, \alpha_{1}$.

Replacing $\frac{1}{4} t$ by $T$, we have

$$
\frac{R\left(x, 4 \alpha_{1} T\right)}{R(y, 3 T)} \leq C_{3} \exp \left(\frac{\rho^{2}}{4}\right)
$$

for $x, y \in B_{4 \alpha_{0} T}(p, \rho \sqrt{T})$ for some constant $C_{2}$ independent of $x, y, t, \rho, \alpha_{0}, \alpha_{1}$. From this the lemma follows.

We will also need the following result, which is basically from [29, 11] (see also [12]).

Lemma 2.2. Let $(M, g)$ be a complete Kähler manifold. Let $p \in M$ and $r_{0}>0$. Suppose $|R m(g)|+|\nabla R m(g)| \leq c$ for some $c$ on $B\left(p, r_{0}\right)$. Then there exist $r>0$ and a constant $C>0$ depending only on $r_{0}, c$ and $n$, and a holomorphic map

$$
\Phi: D(r) \rightarrow M
$$

with the following properties:

(i) $\Phi$ is a local biholomorphism from $D(r) \subset \mathbb{C}^{n}$ onto its image,

(ii) $\Phi(0)=p$,

(iii) $\Phi^{*}(g)(0)=g_{e}$,

(iv) $\frac{1}{C} g_{e} \leq \Phi^{*}(g) \leq C g_{e}$ in $D(r)$,

where $g_{e}$ is the standard metric on $\mathbb{C}^{n}$.

We may now state our first

Theorem 2.1. Suppose that at some $p \in M$ we have $\operatorname{Ric}(p, t) \geq c g(p, t)$ for all $t$ for some $c>0$. Then the following are true.

(i) The injectivity radius of $g(t)$ at $p$ satisfies $\operatorname{inj}_{t}(p) \geq$ a for some $a>0$ for all $t$.

(ii) $M^{n}$ is homeomorphic to the Euclidean space and is biholomorphic to a pseudoconvex domain in $\mathbb{C}^{n}$.

(iii) For any $t_{i} \rightarrow \infty$ we can find a subsequence $t_{i_{k}}$ such that $\left(M, g\left(t+t_{i_{k}}\right), p\right)$ converges to a complete eternal solution $\left(M_{\infty}, g_{\infty}(t), o\right)$ of (2.1) with positive Ricci curvature and bounded nonnegative holomorphic bisectional curvature, and $M_{\infty}$ is biholomorphic to $\mathbb{C}^{n}$.

(iv) For any $t_{i} \rightarrow \infty$, there exist a subsequence $t_{i_{k}}$ and $s_{i_{k}} \geq t_{i_{k}}$ such that $\left(M, g\left(s_{i_{k}}\right), p\right)$ converges to a steady gradient Kähler-Ricci soliton with positive Ricci curvature and bounded nonnegative holomorphic bisectional curvature which is biholomorphic to $\mathbb{C}^{n}$.

Proof. Assume (i) is true. Since the curvature, and its covariant derivatives, of $g(t)$ are uniformly bounded by [28, for each $t \geq 1$ there is a biholomorphism $\Phi_{t}$ from $D(r) \rightarrow M$ satisfying the conditions in Lemma 2.2 with $r$ and $C$ being independent of $t$. Since Ric $>0$ for all $t$, it is easy to see that $\Phi_{t}(D(r))$ exhausts $M$ as $t \rightarrow \infty$ by the eigenvalue inequality for $R c(t)$ in [13] (Theorem 6.1). Hence $M$ is homeomorphic 
to the Euclidean space by [1. This proves the first part of (ii). The second part of (ii) then follows from Theorem 1.2 in [13.

(iii) follows from (i), the standard result on compactness of solutions of Ricci flow 22 and Theorem 1.4 in $[12$.

(iv) will follow from (i) and the content in the proof of (i).

It remains to prove (i). Suppose there exist $t_{i} \rightarrow \infty$ such that $\lim _{i \rightarrow \infty} \operatorname{inj}_{t_{i}}(p)=$ 0 . Since the curvatures are uniformly bounded in space-time, for any positive integer $k \geq 1$ there is a positive constant $C_{1}$ which is independent of $i$, but possibly depending on $k$, such that

$$
\frac{1}{C_{1}} g(t) \leq g\left(t_{i}\right) \leq C_{1} g(t)
$$

for any $t \in\left[t_{i}, t_{i}+k\right]$. By volume comparison and [17] on the injectivity radius bound we have:

$$
\lim _{i \rightarrow \infty} \sup _{t \in\left[t_{i}, t_{i}+k\right]} \operatorname{inj}_{t}(p)=0
$$

for any $k$. On the other hand, since the scalar curvatures of $g(t)$ are uniformly bounded, for any $i$, there exists $s_{i} \in\left[t_{i}, t_{i}+k\right]$ such that the scalar curvature $\left|\frac{\partial}{\partial t} R(p, t)\right|_{t=s_{i}} \mid \leq C_{2} / k$, where $C_{2}=2 \sup _{t} R(p, t)$. By passing to a subsequence still denoted by $t_{i}$, we can find $k_{i} \rightarrow \infty$ and $s_{i} \in\left[t_{i}, t_{i}+k_{i}\right]$ such that

$$
\lim _{i \rightarrow \infty} \operatorname{inj}_{s_{i}}(p)=0,\left.\lim _{i \rightarrow \infty} \frac{\partial}{\partial t} R(p, t)\right|_{t=s_{i}}=0 .
$$

By Lemma 2.2, there is $r>0$ and $C_{3}>0$ independent of $i$ such that for each $i$, there exists a holomorphic map $\Phi_{i}: D(r) \rightarrow M$ corresponding to the metric $g\left(s_{i}\right)$ satisfying the conditions in the lemma with $C=C_{3}$ in property (iv) in the lemma. Now by considering the sequence $\left(D(r), g_{i}(s)=\Phi_{i}^{*} g\left(s_{i}+s\right)\right)$, we may then obtain a local subsequence limit solution $(D(r), h(z, t))$ to (2.1) on an eternal time interval and satisfying $\frac{\partial}{\partial t} R^{h}(0,0)=0$, where $R^{h}$ is the scalar curvature of $h$. By Theorem 2.1 in 12 we find that $(D(r), h(0))$ is a local steady gradient Kähler-Ricci soliton with positive Ricci curvature centered at the origin. Namely, there is a real-valued function $f$ on $D(r)$ such that $R_{i \bar{\jmath}}^{h}=f_{i \bar{\jmath}}$ and $f_{i j}=0$ in $D(r)$, where $R_{i \bar{\jmath}}^{h}$ is the Ricci curvature of $h(0)$. Thus $Z^{i}=f^{i}$ is a holomorphic vector field, and it can be shown that the gradient of $f$ is zero at the origin (see [12 for example), which is an isolated zero because $f_{i \bar{\jmath}}=R_{i \bar{\jmath}}^{h}>0$. In fact by its construction in [12, §2] and [6], there exists $0<r_{1}<r$ such that $Z$ is the unique solution of the equation

$$
R_{, i}+R_{i \bar{j}} Z^{\bar{j}}=0
$$

in $D\left(r_{1}\right)$ relative to the metric $h(0)$.

Note that (2.7) has a unique solution $Z \in T^{(0,1)}$ on any Kähler manifold having positive Ricci curvature. By our assumptions on the positivity of Ricci curvature, we may then let $W(i) \in T^{(0,1)} M$ be the unique solutions to (2.7) on $\left(M, g\left(s_{i}\right)\right)$ for any $k$. In particular, for any $i$, as $\Phi_{i}$ is a local biholomorphism, $V(i)=\left(\Phi_{i}\right)^{*}(W(i))$ is a smooth $(0,1)$ vector field in $D(r)$. By the definition of $g\left(s_{i}\right), V(i)$ is just the unique solution of (2.7) in $D\left(r_{1}\right)$ relative to the metric $\left(\Phi_{i}\right)^{*}\left(g\left(s_{i}\right)\right)$. By uniqueness and the fact that $\left(\Phi_{i}\right)^{*}\left(g\left(s_{i}\right)\right) \rightarrow h$, we conclude that:

$V(i)$ converges to $Z$ uniformly on compact sets in $D\left(r_{1}\right)$ in the $C^{\infty}$ topology. 
It is easy to see that the integral curves of $-Z$ in $D(r)$ will converge to the origin. Since $V(i)$ converge to $Z$, one expects the integral curves of $V(i)$ to have similar behaviors if $i$ is large. In fact, in real coordinates $x_{\alpha}$ of $D(r)$, the integral curves of $-Z$ are given by the following ODE:

$$
x_{\alpha}^{\prime}=-\lambda_{\alpha} x_{\alpha}+F_{\alpha}(x),
$$

where $\lambda_{\alpha} \geq c>0$ are the positive eigenvalues of $R_{i \bar{\jmath}}^{h}$ at the origin, $|F(x)|=\left(|x|^{2}\right)$ and $|d F(x)|=O(|x|)$. On the other hand, for any $\epsilon>0$ there exists $i_{0}$ and $0<r_{1}<r$ such that the integral curves of $V(i)$ for $i \geq i_{0}$ are given by

$$
x_{\alpha}^{\prime}=-\lambda_{\alpha} x_{\alpha}+G_{\alpha}^{i}(x),
$$

where $\left|G^{i}-F\right|+\left|d G^{i}-d F\right| \leq \epsilon$ in $D\left(r_{1}\right)$.

Fix $r_{1}>r_{2}>0$ and suppose $x(\tau)$ is an integral curve of $-V(i)$ in $D(r)$. Then

$$
\begin{aligned}
\frac{d}{d \tau}|x|^{2} & \leq-2 c|x|^{2}+\left|G^{i}(x)\right||x| \\
& \leq-2 c|x|^{2}+\epsilon|x|+C_{4}|x|^{3} \\
& \leq-\frac{3}{2} c|x|^{2}+\epsilon|x|,
\end{aligned}
$$

where $C_{4}$ depends only on $F$, provided $r_{2}<\frac{c}{2 C_{3}}$ and $|x| \leq r_{2}$. Fix $r_{2}$ satisfying these conditions. Then if $\frac{r_{2}}{2} \leq|x| \leq r_{2}$ we have

$$
\frac{d}{d \tau}|x|^{2} \leq-\frac{3}{2} c|x|^{2}+\frac{2 \epsilon}{r}|x|^{2}<0
$$

if $\epsilon>0$ is small enough. Choose such an $\epsilon$. Hence for $i \geq i_{0}$ large enough any integral curve of $V(i)$ starting in $D\left(r_{2}\right)$ will stay inside $D\left(r_{2}\right)$.

Now let $x(\tau)$ and $y(\tau)$ be two integral curves of $V(i)$ inside $D\left(r_{2}\right)$. Then

$$
\begin{aligned}
\frac{d}{d \tau}|x-y|^{2} & =-2 \sum_{\alpha} \lambda_{\alpha}\left(x_{\alpha}-y_{\alpha}\right)^{2}+\sum_{\alpha}\left(G_{\alpha}^{i}(x)-G_{\alpha}^{i}(y)\right)\left(x_{\alpha}-y_{\alpha}\right) \\
& \leq-2 c|x-y|^{2}+\sum_{\alpha}\left\|d G^{i}\left(z_{\alpha}\right)\right\|\left(x_{\alpha}-y_{\alpha}\right)^{2} \\
& \leq-2 c|x-y|^{2}+\epsilon|x-y|^{2}+\sum_{\alpha}\left\|d F\left(z_{\alpha}\right)\right\|\left(x_{\alpha}-y_{\alpha}\right)^{2} \\
& \leq-c|x-y|^{2}
\end{aligned}
$$

for some $z_{\alpha}$ inside $D\left(r_{2}\right)$, provided $r_{2}$ and $\epsilon$ are sufficiently small, both depending only on the constant $c$. Hence

$$
|x-y|^{2}(\tau) \leq \exp (-c \tau)|x-y|^{2}(0) \leq 4 r^{2} \exp (-c \tau)
$$

If $x(\tau)$ is an integral curve in $D\left(r_{2}\right)$, and if $\tau_{2}>\tau_{1}>0$, then

$$
y(\tau)=x\left(\tau_{2}-\tau_{1}+\tau\right)
$$

is also an integral curve in $D\left(r_{2}\right)$. Hence $\left|x\left(\tau_{1}\right)-x\left(\tau_{2}\right)\right|^{2} \leq 4 r^{2} \exp \left(-c \tau_{1}\right)$. Hence $x(\tau)$ will converge to a point $x_{0} \in \overline{D\left(r_{2}\right)}$, and by (2.12), we see that any set in $D\left(r_{2}\right)$ will contract to $x_{0}$ along integral curves. In fact, $x_{0} \in \overline{D\left(\frac{r_{2}}{2}\right)}$ by (2.11). Moreover, $x_{0}$ is a fixed point of $V(i)$.

We now claim that $\Phi_{i}$ is injective on $D\left(r_{2}\right)$ for $i \geq i_{0}$. Assume this is not the case and that for some $z_{1} \neq z_{2} \in D\left(r_{2}\right)$ we have $\Phi_{i}\left(z_{1}\right)=\Phi_{i}\left(z_{2}\right)=q \in M$. Let $\gamma_{1}$ and $\gamma_{2}$ be two integral curves for $V(i)$ starting at $z_{1}$ and $z_{2}$, respectively. Then by the 
construction of $V(i), \Phi_{i}\left(\gamma_{1}\right)$ and $\Phi_{i}\left(\gamma_{2}\right)$ are integral curves for $W(i)$ starting from $q$. Hence by the uniqueness of integral curves we must have $\Phi_{i}\left(\gamma_{1}(\tau)\right)=\Phi_{i}\left(\gamma_{2}(\tau)\right)$ for all $\tau$. On the other hand, for all $\tau$, we have $\gamma_{1}(\tau) \neq \gamma_{2}(\tau)$ also by uniqueness of integral curves. But $\gamma_{1}(\tau)$ and $\gamma_{2}(\tau)$ both converge to an $x_{0} \in \bar{D}\left(r_{2}\right)$. It is readily seen that these facts contradict the fact that $\Phi_{i}$ is a biholomorphism in some neighborhood of $x_{0}$. Thus we have shown that $\Phi_{i}$ is injective on $D\left(r_{2}\right)$ for all $i \geq i_{0}$.

Now it is easy to see that $\Phi_{i}\left(D\left(r_{2}\right)\right)$ is contained in some geodesic ball $B_{s_{i}}\left(p, r_{3}\right)$ for some $r_{3}$ independent of $i$. Thus by the injectivity of $\Phi_{i}$ shown above, and by the fact that $\Phi_{i}^{*}\left(g\left(s_{i}\right)\right)$ is uniformly equivalent to the Euclidean metric by Lemma 2.2, it is easy to see that there exists $b>0$ such that for $i \geq i_{0}$ large enough, $B_{s_{i}}\left(p, r_{3}\right)$ has volume bounded below by $b$. By the result of Cheeger-Gromov-Taylor [17, $\operatorname{inj}_{s_{i}}(p)$ must be uniformly bounded away from zero. But this contradicts our assumption that $\operatorname{inj}_{s_{i}}(p) \rightarrow 0$. This completes the proof of the theorem by contradiction.

Next we consider the case that $t R(p, t)$ is uniformly bounded.

Theorem 2.2. Suppose that at some $p \in M$ we have $t R(p, t) \leq c$ for all $t$ and some $c>0$. Then the following are true.

(i) The injectivity radius of $g(t)$ at $p$ satisfies $\operatorname{inj}_{t}(p) \geq a t^{\frac{1}{2}}$ for some $a>0$ for all $t$.

(ii) $M^{n}$ is biholomorphic to $\mathbb{C}^{n}$.

(iii) Let $\tilde{g}_{i \bar{\jmath}}(s)=e^{-s} g\left(e^{s}\right)$. Then for any $s_{i} \rightarrow \infty$ we can find a subsequence $s_{i_{k}}$ such that $\left(M, \tilde{g}_{i \bar{\jmath}}\left(s_{i_{k}}\right), p\right)$ converges to an expanding gradient Kähler-Ricci soliton with positive Ricci curvature which is biholmorphic to $\mathbb{C}^{n}$.

We will need the following lemma.

Lemma 2.3. With the same assumptions as in Theorem 2.2, for any $\rho>0$ and integer $k \geq 0$, there is a constant $C$ such that

$$
\left\|\nabla^{k} \operatorname{Rm}(x, t)\right\|_{t}^{2} \leq \frac{C}{t^{2+k}}
$$

for all $x \in B_{t}(p, \rho \sqrt{t})$ and for all $t>0$, where $\nabla$ and the norm are taken with respect to $g(t)$.

Proof. By the assumptions and Lemma 2.1, there exists a constant $C_{1}$ such that for all $T>0$ and $r>0$,

$$
\frac{R(x, t)}{R(p, 3 T)} \leq C_{1} \exp \left(\frac{r^{2}}{4}\right)
$$

for all $x \in B_{T}(p, r \sqrt{T})$ and $T \leq t \leq 2 T$. Consider the metrics

$$
\hat{g}(s)=\frac{1}{T} g(T+s T) .
$$

Then $\hat{g}$ satisifies the Kähler-Ricci flow equation with nonnegative holomorphic bisectional curvature. By the assumption that $t R(p, t)$ is uniformly bounded and (2.14) and the fact that $\hat{g}(s)$ has nonnegative holomorphic bisectional curvature, we have

$$
\|\widehat{R} m\| \leq C_{2} \exp \left(\frac{r^{2}}{4}\right)
$$


in $\widehat{B}_{0}(p, r) \times[0,2]$, where $C_{2}$ is a constant independent of $r$ and $T$ and where $\widehat{\mathrm{Rm}}$, $\|\cdot\|$ are the curvature tensor and the norm with respect to $\hat{g}$. Thus by the local derivatives estimates of Shi [26], see also [20], for any integer $k \geq 0$, there is a constant $C_{3}$ which is independent of $t$ such that

$$
\left\|\widehat{\nabla}^{k} \widehat{\mathrm{Rm}}\right\| \leq C_{3}
$$

in $\hat{B}_{0}\left(p, \frac{r}{2}\right) \times\{1\}$.

On the other hand, by the Kähler-Ricci flow equation, we conclude that

$$
C_{4} \exp \left(\exp \left(\frac{r^{2}}{4}\right)\right) \hat{g}(0) \leq \hat{g}(1) \leq \hat{g}(0)
$$

in $\widehat{B}_{0}(p, r)$ for some positive constant $C_{4}$ independent of $r$ and $T$. Hence we have $\widehat{B}_{1}(p, \rho) \subset \widehat{B}_{0}\left(p, \frac{r}{2}\right)$, where $\rho=C_{4}^{\frac{1}{2}} \exp \left(\frac{1}{2} \exp \left(\frac{r^{2}}{4}\right)\right) \frac{r}{2}$.

Given $\rho>0$, let $r$ be such that $\rho=C_{4}^{\frac{1}{2}} \exp \left(\frac{1}{2} \exp \left(\frac{r^{2}}{4}\right)\right) \frac{r}{2}$, rescale $\hat{g}$ back to $g$, and conclude from (2.16) that the lemma is true.

Now we are ready to prove the theorem.

Proof of Theorem 2.2. To prove (i), let $\tilde{g}_{i \bar{\jmath}}$ be as in (iii). Then $\tilde{g}_{i \bar{\jmath}}(s)$ solves the normalized Kähler-Ricci flow equation:

$$
\frac{\partial \widetilde{g}_{i \bar{\jmath}}}{\partial t}=-\widetilde{R}_{i \bar{\jmath}}-\widetilde{g}_{i \bar{\jmath}}
$$

on $M \times(-\infty, \infty)$. By Lemma 2.3, the fact that $\tilde{g}_{i \bar{\jmath}}$ has positive Ricci curvature, and the fact that the eigenvalues of $\widetilde{\text { Ric }}$ are nondecreasing by Theorem 6.1 in [13, we can proceed exactly as in the proof of Theorem 2.1 (i) to show that the injectivity radius of $\tilde{g}_{i \bar{\jmath}}(s)$ at $p$ is uniformly bounded below away from zero 2 Rescaling back to $g(t)$, we see that (i) is true. Note that here we use the fact that if we take a limit along a sequence $s_{i}$ as in the proof of Theorem 2.1(i), then in the limit metric, the scalar curvature at the origin is constant in time.

Using Lemma 2.3 and (i), the proof of Theorem 1.1 in 11] can be carried over to prove that $M$ is biholomorphic to $\mathbb{C}^{n} 3$

Since $\tilde{g}_{i \bar{\jmath}}(s)$ is decreasing, for any $\rho>0$ and $s_{0} \geq 0$, the curvature together with its derivatives is uniformly bounded on $B_{s_{0}}(p, \rho)$ for all $s \geq s_{0}$. Combining with (i), one can apply the proof of the local version [19, Corollary 3.18] of compactness of solutions of Hamilton [22] to conclude that for any $s_{i} \rightarrow \infty$ we can find a subsequence $s_{i_{k}}$ such that $\left(M, \tilde{g}_{i \bar{\jmath}}\left(s+s_{i_{k}}\right), p\right)$ converges to a complete eternal solution of (2.17) which is in fact an expanding gradient Kähler-Ricci soliton with positive Ricci curvature and nonnegative holomorphic bisectional curvature. The fact that the limit solution is an expanding gradient Kähler-Ricci soliton can be proved as in [12, §2]. The fact that the limiting manifold is biholomorphic to $\mathbb{C}^{n}$ follows from [10, 2.

Remark 2.1. Theorem 2.2 was proved by the authors in 12 under the stronger assumption that $t R(p, t)$ is uniformly bounded for all $t \geq 0$ independently of $p$. In particular, it was proved there that $M$ is biholomorphic to $\mathbb{C}^{n}$. We point out that

\footnotetext{
${ }^{2}$ In this case the local limit $(D(r), h(0))$ will be a local expanding gradient Kähler Ricci soliton.

${ }^{3}$ In Theorem 1.1 of [1], there exists a long-time solution to Kähler-Ricci flow such that the estimate in Lemma 2.3 holds for all $x \in M$. However, only the precise statement of Lemma 2.3 was actually used in the proof of the theorem.
} 
the injectivity radius bound in Theorem 2.2 (i) allows a direct application of the methods in [11, and thus a more direct proof of this result.

By the result of [4], if $t R(p, t)$ is not bounded, then $t R(p, t) \rightarrow \infty$ as $t \rightarrow \infty$. We consider this case in the next theorem.

Theorem 2.3. Suppose that at some $p \in M$, $\lim _{t \rightarrow \infty} t R(p, t)=\infty$ and $\inf _{t} R(p, t)$ $=0$. Suppose also that the eigenvalues of $\operatorname{Ric}(p, t)$ are uniformly pinched, i.e. $R_{i \bar{\jmath}} \geq c R g_{i \bar{\jmath}}$ at $(p, t)$ for all $t$ for some $c>0$. Then there exists $t_{i} \rightarrow \infty$ and $a_{i}>0$ with $\lim _{i \rightarrow \infty} a_{i}=0$ such that the following are true.

(i) The injectivity radius of $g\left(t_{i}\right)$ at $p$ satisfies $\operatorname{inj}_{t_{i}}(p) \geq c a_{i}^{-1}$ for some $c>0$.

(ii) $M^{n}$ is homeomorphic to Euclidean space and is biholomorphic to a pseudoconvex domain in $\mathbb{C}^{n}$.

(iii) $\left(M^{n}, a_{i} g\left(t_{i}\right), p\right)$ converge to a steady gradient Kähler-Ricci soliton with positive Ricci curvature which is biholomorphic to $\mathbb{C}^{n}$.

Proof. To prove (i), since $\inf _{t} R(p, t)=0$ and Ric $>0$ for all $t$, we can find $T_{i} \rightarrow \infty$ such that $a_{i}=R\left(p, 3 T_{i}\right)=\inf _{t \in\left[0,3 T_{i}\right]} R(p, t)$. Then $a_{i}>0$ and $\lim _{i \rightarrow \infty} a_{i}=0$. Now the metrics $g_{i}(t)=a_{i} g\left(T_{i}+\frac{t}{a_{i}}\right)$ solve (2.1) for $-a_{i} T_{i} \leq t<\infty$. Moreover, by the choice of $T_{i}$ we have $R^{(i)}(p, t) \geq 1$ for $-a_{i} T_{i} \leq t \leq 2 a_{i} T_{i}$, where $R^{(i)}$ is the scalar curvature of $g_{i}$. On the other hand, by Lemma 2.1, there is a constant $C_{1}$ independent of $i$ such that $R^{(i)}(p, t) \leq C_{1}$ for all $0 \leq t \leq 2 a_{i} T_{i}$. Hence there is $0 \leq s_{i} \leq \frac{1}{4} a_{i} T_{i}$ such that $\left|\frac{\partial}{\partial t} R^{(i)}\left(p, s_{i}\right)\right| \leq 8 C_{1} /\left(a_{i} T_{i}\right)$. By the assumption that $t R(p, t) \rightarrow \infty$ as $t \rightarrow \infty$, we conclude that $\lim _{i \rightarrow \infty} a_{i} T_{i}=\infty$ and

$$
\lim _{i \rightarrow \infty} \frac{\partial}{\partial t} R^{(i)}\left(p, s_{i}\right)=0 \text {. }
$$

We now consider the sequence of metrics $h_{i}(t)=g_{i}\left(s_{i}+t\right)$ which solve (2.1) for $-a_{i} T_{i}-s_{i} \leq t<\infty$. By Lemma 2.1 and the definition of $a_{i}$, there is a constant $C_{2}$ independent of $i$ such that

$$
\widehat{R}^{(i)}(x, t) \leq C_{2}
$$

for all $x \in \widehat{B}_{0}^{(i)}\left(p, \sqrt{a_{i} T_{i}}\right)$ and $0 \leq t \leq \frac{1}{2} a_{i} T_{i}$, where $\widehat{R}^{(i)}(x, t)$ is the scalar curvature of $h_{i}(t)$ and $\widehat{B}_{r}^{(i)}$ is the geodesic ball with respect to $h^{(i)}$.

Since the eigenvalues of the Ricci curvature of $g(t)$ at $p$ are uniformly pinched, the Ricci curvature of $h^{i}(p, 0)$ is uniformly bounded from below. Noting that $a_{i} T_{i} \rightarrow \infty$ as $i \rightarrow \infty$, by (2.18) and (2.19), we can proceed as in the proof of Theorem 2.1 (i) to show that the injectivity radius of $h^{i}(0)$ is uniformly bounded from below. Rescaling back to $g(t)$, using the definition of $a_{i}$, we see that (i) is true.

Part (ii) follows from part (i) as in the proof of Theorem 2.1

By the above facts, in particular that (2.18) holds on a sequence of balls with radii going to $\infty$, one can proceed as in the proof of Theorem 2.1 (iii) to conclude that $\left(M, h^{i}(0), p\right)$ converges to a complete steady gradient Kähler-Ricci soliton with positive Ricci curvature and nonnegative holomorphic bisectional curvature. Note that the gradient of the function defining the gradient Kähler-Ricci soliton has a unique zero by (2.18) and the fact that the Ricci curvature is positive. Hence the gradient Kähler-Ricci soliton is biholomorphic to $\mathbb{C}^{n}$ by the results in 10, 2 .

The methods of proof in the theorems above allow us to study the holomorphic isometries of $(M, g(0))$. 
Theorem 2.4. Let $(M, g(t))$ be as in the BASIC Assumption 1. Suppose in addition that $t R(p, t) \rightarrow \infty$ and the eigenvalues of the $R c(p, t)$ are uniformly pinched. Let $\Gamma$ be a finite subset of the holomorphic isometry group of $(M, g(0))$. Then there exists $q \in M$ such that $q$ is fixed by any $\gamma \in \Gamma$.

Proof. By Theorems 2.1 and 2.3 we can find $t_{i} \rightarrow \infty$ and a bounded sequence $a_{i}>0$ such that $\left(M, a_{i} g\left(t_{i}\right), p\right)$ converge to a steady gradient Kähler-Ricci soliton with positive Ricci curvature. By the proof of Theorem 2.1, we conclude that there exists $a>0$ such that $\nabla_{i} R^{(i)}$ has a unique zero at $q_{i} \in B_{i}(p, a)$ which lies inside $B_{i}\left(p, \frac{a}{3}\right)$. Here $B_{i}$ is the geodesic ball, $\nabla_{i}$ is the covariant derivative and $R^{(i)}$ is the scalar curvature with respect to $a_{i} g\left(t_{i}\right)$.

We claim that for any holomorphic isometry $\gamma$ of $(M, g(0))$, there is $i_{0}$ such that $\gamma\left(q_{i}\right)=q_{i}$ for all $i \geq i_{0}$. It is easy to see that the theorem follows from this claim as $\Gamma$ is finite.

By the uniqueness of Kähler-Ricci flow [18 (see also 21]), $\gamma$ is also an isometry of $g(t)$ and hence of $a_{i} g\left(t_{i}\right)$ for all $i$. So $\gamma\left(q_{i}\right)$ is also a zero of $\nabla_{i} R^{(i)}$. Since $q_{i}$ is the unique zero of $\nabla_{i} R^{(i)}$ in $B_{i}(p, a)$, it is easy to see that the claim is true if $\gamma$ fixes $p$.

Suppose $\gamma(p) \neq p$. Since for any $\epsilon>0, B_{t_{i}}(p, \epsilon)$ exhausts $M$, see [13], and since $a_{i}$ is uniformly bounded, we conclude that there exists $i_{0}$ such that if $i \geq i_{0}$, then $\gamma(p) \in B_{i}\left(p, \frac{a}{3}\right)$. Hence $\gamma\left(q_{i}\right) \in B_{i}(p, a)$. Since $q_{i}$ is the unique zero of $\nabla_{i} R^{(i)}$ in $B_{i}(p, a)$, we have $\gamma\left(q_{i}\right)=q_{i}$ for $i \geq i_{0}$.

In case $t R(p, t)$ is uniformly bounded in $t$ or if $g(t)$ is an eternal solution, then we have the following much stronger result.

Theorem 2.5. Let $(M, g(t))$ be as in the BAsic Assumption 1. Suppose in addition that either (i) for some $p \in M, t R(p, t)$ is uniformly bounded in $t$, or (ii) $g(t)$ is an eternal solution. Then there exists $q \in M$ such that $q$ is fixed by any holomorphic isometry of $(M, g(0))$.

Proof. First consider the case that $g(t)$ is an eternal solution. Then by the monotonicity of the eigenvalues of the Ricci curvature in $t$ [13] (Theorem 6.1), the Ricci curvature at $p$ is uniformly bounded below away from zero for all $t$. On the other hand, by the LYH type differential inequality by Cao [6], we know that $\frac{d}{d t} R(p, t) \geq 0$. Hence as in [12], for any $t_{i} \rightarrow \infty,\left(M, g\left(t_{i}\right), p\right)$ will subconverge to a gradient Kähler-Ricci soliton with positive Ricci curvature and nonnegative holomorphic bisectional curvature. Hence as in the proof of Theorem 2.4, we can prove that there exist $T>0$ and some $a>0$ such that for $t \geq T$ there exists $q(t) \in B_{t}(p, a)$ which is the unique point satisfying $\nabla_{t} R(q(t), t)=0$ in $B_{t}(p, 3 a)$.

Also as in the proof of Theorem 2.4 we can prove that for every holomorphic isometry $\gamma$, there exists $T_{\gamma}$ such that $\gamma(q(t))=q(t)$ for all $t \geq T_{\gamma}$.

We want to prove that every isometry $\gamma$ fixes $q(T)$. Suppose $\gamma(q(T)) \neq q(T)$. Since $d_{T}(p, q(T))<a$ by uniqueness of $q(T)$, we must have $d_{T}(\gamma(q(T)), q(T)) \geq 2 a$. On the other hand, we have

$$
d_{T_{\gamma}}\left(\gamma\left(q\left(T_{\gamma}\right)\right), q\left(T_{\gamma}\right)\right)=0
$$

and $T<T_{\gamma}$ by the choice of $T_{\gamma}$. Now by uniqueness of $q(t)$ and the fact that $g(t)$ is a solution of the Kähler-Ricci flow equation, it is not hard to see that $q(t)$ describes a continuous path on $M$ with respect to $t$, and thus the same is true for $\gamma(q(t))$. It follows then, that for some $T<T_{0}<T_{\gamma}$, we must have $d_{T_{0}}\left(\gamma\left(q\left(T_{0}\right)\right), q\left(T_{0}\right)\right)=a$. 
But this would violate the fact that $q\left(T_{0}\right)$ is the unique zero of $\nabla_{T_{0}} R$ in $B_{T_{0}}(p, 3 a)$. This proves the case when $g(t)$ is an eternal solution.

The case that $t R(p, t)$ is uniformly bounded in $t$ can be proved similarly using Theorem 2.2 .

By the results of Shi 28] (see also [12]), if $(M, g)$ has bounded nonnegative holomorphic bisectional curvature with average quadratic curvature decay and positive Ricci curvature somewhere on $M$, then there exists a long-time solution to the Kähler-Ricci flow such that $t R(p, t)$ is uniformly bounded independently of $p$. We thus have the following immediate corollary of Theorems 2.2 and 2.5 .

Corollary 2.1. Let $(M, g)$ be a complete noncompact Kähler manifold with nonnegative holomorphic bisectional curvature. Suppose $k(x, r) \leq C /\left(1+r^{2}\right)$ for some $C$ and all $r$ and $x$, and that $R c(p)>0$ for some $p \in M$. Then $M$ is biholomorphic to $\mathbb{C}^{n}$, and there exists $q \in M$ such that $q$ is fixed by any holomorphic isometry of $(M, g)$.

\section{Fiber bundle struCtures}

In this section we study the structure of a complete noncompact Kähler manifold with nonnegative holomorphic bisectional curvature using the Kähler-Ricci flow. To motivate our discussion, let us consider an example. Take a complete noncompact Kähler metric $h$ on $\mathbb{C}^{n}$ with positive holomorphic bisectional curvature which is $U(n)$-invariant; see [5, 6, 31]. Let $\tilde{M}=\mathbb{C}^{l} \times \mathbb{C}^{n}\left(l \geq 1\right.$ with metric $\widetilde{g}=g_{e} \times h$, where $g_{e}$ is the standard metric on $\left.\mathbb{C}^{l}\right)$. Let $k$ be a nonzero real number. Define $\gamma: \tilde{M} \rightarrow \tilde{M}$ as $\gamma(\zeta, z)=\left(\zeta_{1}+k, \zeta_{2}, \ldots, \zeta_{l}, e^{2 \pi k \sqrt{-1}} z\right)$, where $\zeta \in \mathbb{C}^{l}$ and $z \in \mathbb{C}^{n}$. Then $\gamma$ is a holomorphic isometry of $(\tilde{M}, \widetilde{g})$ and $\gamma^{m}$ has no fixed point unless $m=0$. Let $\Gamma$ be the cyclic group generated by $\gamma$. Then $(M, g)=(\tilde{M}, \widetilde{g}) / \Gamma$ is a complete noncompact manifold with nonnegative holomorphic bisectional curvature, which is a vector bundle over $\mathbb{C}^{l} / \Gamma^{\prime}$, where $\Gamma^{\prime}$ is the group generated by $\gamma^{\prime}$ which sends $\left(\zeta_{1}, \zeta_{2}, \ldots, \zeta_{l}\right)$ to $\left(\zeta_{1}+k, \zeta_{2}, \ldots, \zeta_{l}\right)$.

Now let $(M, g(t))$ be a long-time solution of the Kähler-Ricci flow (2.1) on a complete noncompact Kähler manifold with bounded nonnegative holomorphic bisectional curvature yet without any assumption on the positivity of the Ricci curvature. If $\left(\tilde{M}, \tilde{g}_{i \bar{\jmath}}(t)\right)$ is the universal cover of $M$ with corresponding lift $\tilde{g}_{i \bar{j}}(t)$, then $\tilde{g}_{i \bar{\jmath}}(t)$ is also such a solution to Kähler-Ricci flow. As mentioned in the introduction, $\left(\tilde{M}, \tilde{g}_{i \bar{\jmath}}(t)\right)$ splits for each $t>0$ as $\left(\mathbb{C}^{k} \times N, g_{e} \times h(t)\right)$, where $g_{e}$ is the standard Euclidean metric on $\mathbb{C}^{k}$ and $h(t)$ solves (1.2) and has positive Ricci curvature for $t>0$. We will use our results from $\S 2$, for the factor $(N, h(t))$, together with the arguments in [25, §5] to provide a fiber bundle description of $M$. In Corollary 3.1 we treat the particular case of an initial complete noncompact Kähler manifold having bounded nonnegative holomorphic bisectional curvature which decays quadratically in the average sense.

We begin with the following

BASIC ASSUMPTION 2: Let $M^{n}$ be a noncompact complex manifold and let $\left(M^{n}, g(t)\right)$ be a complete solution of the Kähler-Ricci flow

$$
\frac{\partial g_{i \bar{\jmath}}}{\partial t}=-R_{i \bar{\jmath}}
$$


on $M \times[0, \infty)$ with uniformly bounded nonnegative holomorphic bisectional curvature in space-time, so that one of the following is true:

Case 1: For some $p \in M$ (and hence for all points), $t R(p, t)$ is uniformly bounded in $t$.

Case 2: For some point $p \in M$, $\lim _{t \rightarrow \infty} t R(p, t)=\infty$ and the positive eigenvalues of $\operatorname{Ric}(t)$ at $p$ are uniformly pinched for all $t$.

Theorem 3.1. With the BAsic Assumption 2, there is $k \geq 0$ such that for all t, $(M, g(t))$ is a holomorphic Riemannian fiber bundle over $\mathbb{C}^{k} / \Gamma$, where $\Gamma$ is a discrete subgroup of holomorphic isometries of $\mathbb{C}^{k}$, with fiber being $\mathbb{C}^{n-k}$ in Case 1 and a pseudoconvex domain in $\mathbb{C}^{n-k}$ in Case 2. If in Case 2, $g(t)$ is in fact an eternal solution, then the fiber is also $\mathbb{C}^{n-k}$.

Proof. Let $\tilde{M}$ be the universal cover of $M$. By the splitting result of Cao [7, we have $\tilde{M}=\mathbb{C}^{k} \times \tilde{N}$ and $\tilde{g}(t)=g_{\epsilon}(t) \times \tilde{h}(t)$, where $g_{\epsilon}(t)$ is flat and $\tilde{h}(t)$ satisfies the Basic Assumption 1 of $\S 1$ on $\tilde{N}$. In particular, in Case $1, \tilde{N}$ is biholomorphic to $\mathbb{C}^{n-k}$ by Theorem 2.2, and in Case 2, $\tilde{N}$ is homeomorphic to the Euclidean space and biholomorphic to a pseudoconvex domain in $\mathbb{C}^{n-k}$ by Theorems 2.1 and 2.3 . Moreover, if in Case 2, g(t) and hence $\tilde{h}(t)$ is in fact an eternal solution, then by [12, $\tilde{N}$ is biholomorphic to $\mathbb{C}^{n}$.

Let $F \in \Gamma$, the first fundamental group of $M$ with respect to the metric $g(0)$. By the uniqueness of the Kähler-Ricci flow (see [18, 21]), $F$ is a holomorphic isometry with respect to $g(t)$ for all $t$. As a mapping on $\tilde{M}, F(\tilde{x}, \tilde{y})=\left(f_{1}(\tilde{x}, \tilde{y}), f_{2}(\tilde{x}, \tilde{y})\right)$, where $f_{1}: \tilde{M} \rightarrow \mathbb{C}^{k}, f_{2}: \tilde{M} \rightarrow \tilde{N}, \tilde{x} \in \mathbb{C}^{k}$ and $\tilde{y} \in \tilde{N}$.

The following argument is basically as in [25, §5], using the fact that $\tilde{N}$ is biholomorphic either to $\mathbb{C}^{n-k}$ or to a pseudoconvex domain in $\mathbb{C}^{n-k}$ which is homeomorphic to $R^{2(n-k)}$.

We first prove that $f_{2}$ is independent of $\tilde{x}$. Suppose there is $\tilde{x}_{1} \neq \tilde{x}_{2}$ and $\tilde{y}$ such that $f_{2}\left(\tilde{x}_{1}, \tilde{y}\right) \neq f_{2}\left(\tilde{x}_{2}, \tilde{y}\right)$. Let $\alpha$ be a line passing through $\tilde{x}_{1}$ and $\tilde{x}_{2}$ in $\mathbb{C}^{k}$. Then $\alpha(s)=(\gamma(s), \tilde{y})$ is a line in $\tilde{M}$. That is to say, $\alpha$ is a geodesic which is minimizing between any two points in $\alpha$. Hence $F \circ \alpha(s)$ is a line of $\tilde{M}$ too. So its projection onto $\tilde{N}$ is minimizing between any two end points as a curve in $\tilde{N}$. But the projection is $f_{2} \circ \alpha$, which is not a constant curve because $f_{2}\left(\tilde{x}_{1}, \tilde{y}\right) \neq f_{2}\left(\tilde{x}_{2}, \tilde{y}\right)$. Hence it must be a line in $\tilde{N}$. But this implies that a factor $\mathbb{C}$ is split from $\tilde{N}$ by [15. This is impossible because $\tilde{N}$ has positive Ricci curvature. Thus the claim is established.

Next we want to prove that $f_{1}$ is independent of $\tilde{y}$. First note that $F^{-1}=$ $\left(h_{1}, h_{2}\right)$ with $h_{2}$ independent of $\tilde{x}$. Hence $f_{2} \circ h_{2}$ is the identity and $h_{2}=f_{2}^{-1}$. On the other hand, since $F$ is an isometry, $f_{2}$ cannot increase length. For if $\gamma(s)$ is a curve in $\tilde{N}$, then the length for the curve $\alpha(s)=(\tilde{x}, \gamma(s))$ and the length of $F \circ \alpha(s)=\left(f_{1}(\tilde{x}, \gamma(s)), f_{2}(\gamma(s))\right)$ are equal. But the length of $\alpha$ is the same as the length of $\gamma$. So the length of $f_{2}(\gamma(s))$ cannot be larger than the length of $\gamma$. But this is also true for $f_{2}^{-1}$. Hence $f_{2}$ is an isometry. If this is the case, then $f_{1}(\tilde{x}, \gamma(s))$ must be a point. Hence $f_{1}$ is independent of $\tilde{y}$.

Let $\Lambda_{1}=\left\{f_{1} \mid\left(f_{1}, f_{2}\right) \in \Gamma\right.$ for some $\left.f_{2}\right\}$. Then $\Lambda_{1}$ is a subgroup of the holomorphic isometries of $\left(\mathbb{C}^{k}, g_{\epsilon}(t)\right)$ for all $t$. We claim that $\Lambda_{1}$ is fixed point free. Suppose there is $F^{\prime}=\left(f_{1}^{\prime}, f_{2}^{\prime}\right) \in \Gamma$ such that $f_{1}^{\prime}\left(\tilde{x}_{0}\right)=\tilde{x}_{0}$ for some $\tilde{x}_{0}$. Let

$$
G=\left\{f_{2} \in \mid F=\left(f_{1}, f_{2}\right) \in \Gamma \text { for some } f_{1} \text { with } f_{1}\left(\tilde{x}_{0}\right)=\tilde{x}_{0}\right\} .
$$


Then $G$ is a subgroup of the group of the holomorphic isometries of $\tilde{N}$. $G$ acts on $\tilde{N}$ freely because $\Gamma$ acts on $\tilde{M}$ freely. Now $\tilde{h}$ descends to $\tilde{N} / G$ while still satisfying the BAsic Assumption 1 in $\S 1$, and thus must be simply connected by Theorems 2.1, 2.2 and 2.3, which leads to a contradiction if $G$ is not trivial. Thus $f_{1}^{\prime}$ must be trivial and $\Lambda_{1}$ is fixed point free.

Let $\beta$ be the homomorphism from $\Gamma$ to $\Lambda_{1}$ defined by $\beta(F)=f_{1}$, for $F=\left(f_{1}, f_{2}\right)$. Suppose $F=\left(f_{1}^{\prime}, f_{2}^{\prime}\right) \in \Gamma$ and $f_{1}^{\prime}$ is the identity map. Then by a similar argument, we can prove that the group

$$
\left\{f_{2} \mid\left(f_{1}^{\prime}, f_{2}\right) \in \Gamma\right\}
$$

is trivial. Hence $\beta$ is an isomorphism. Then $\Lambda_{1}$ acts on $\tilde{M}$ isometrically holomorphically and freely by $f(\tilde{x}, \tilde{y})=\left(f(\tilde{x}), \rho_{2} \circ \beta^{-1}(f)(\tilde{y})\right)$, where $\rho_{2}$ is the homomorphism given by mapping $F=\left(f_{1}, f_{2}\right) \in \Gamma$ to $f_{2}$.

Hence as shown in [25, §5], $M$ is a Riemannian and holomorphic fiber bundle over $\mathbb{C}^{k} / \Lambda_{1}$ with fiber $\tilde{N}$. By the comments in the first paragraph of the proof, we see that this completes the proof of the theorem.

Theorem 3.2. If $n-k=1$ in the previous theorem, then $(M, g(t))$ is a holomorphic Riemannian line bundle over $\mathbb{C}^{k} / \Gamma$.

Proof. Let $\Gamma$ and $\tilde{N}$ be as in the proof of the previous theorem. Then $\tilde{N}$ is biholomorphic to $\mathbb{C}$ and has a global holomorphic coordinate $z$. Thus for any $F \in \Gamma$ with $F=\left(f_{1}, f_{2}\right), f_{2}(z)=a z+b$ for some $a \neq 0$ and $b \in \mathbb{C}$. Now by Theorem 2.4 any $f_{2}$ has a fixed point. It follows that any nontrivial $f_{2}$ with $F=\left(f_{1}, f_{2}\right) \in \Gamma$ has a unique fixed point. By Theorem 2.4 again, we conclude that there is $z_{0} \in \tilde{N}=\mathbb{C}$ such that $f_{2}\left(z_{0}\right)=z_{0}$ for any holomorphic isometry $f_{2}$ with $F=\left(f_{1}, f_{2}\right) \in \Gamma$. We see that we can choose a global holomorphic coordinate $\tilde{z}$ on $\tilde{N}=\mathbb{C}$ such that in this coordinate, every such $f_{2}$ has the form $f_{2}(\tilde{z})=a \tilde{z}$ for some complex constant $a$. Thus in this case, the fiber bundle structure constructed in the proof of Theorem 3.1 is in fact a line bundle structure. This completes the proof of the theorem.

By the remarks preceding Corollary 2.1, we may have the following immediate corollary to Theorems 3.1 and 3.2

Corollary 3.1. Let $(M, g)$ be a complete noncompact Kähler manifold with nonnegative holomorphic bisectional curvature. Suppose $k(x, r) \leq C /\left(1+r^{2}\right)$ for some $C$ and all $r$ and $x$. Then there is $k \geq 0$ such that $(M, g)$ is a holomorphic Riemannian fiber bundle over $\mathbb{C}^{k} / \Gamma$, where $\Gamma$ is a discrete subgroup of holomorphic isometries of $\mathbb{C}^{k}$, with fiber being $\mathbb{C}^{n-k}$.

Moreover, if $n-k=1$, then the above fiber bundle is in fact a line bundle.

\section{Gradient KÄHleR-Ricci solitons}

In this section we refine the results in the previous section in the case of a steady gradient Kähler-Ricci soliton with bounded and nonnegative holomorphic bisectional curvature. We remark that in the case of an expanding gradient KählerRicci soliton satisfying the same curvature conditions, we have $M$ biholomorphic to $\mathbb{C}^{n}$ by [10]; see also [2].

First let us fix some notation. Let $\left(M^{n}, g\right)$ be a complete steady gradient KählerRicci soliton with bounded and nonnegative holomorphic bisectional curvature. 
Namely, there is a real-valued function $f$ such that

$$
\begin{aligned}
& f_{i \bar{\jmath}}=R_{i \bar{\jmath}}, \\
& f_{i j}=0 .
\end{aligned}
$$

Let $(\tilde{M}, \widetilde{g})$ be the universal cover of $\left(M^{n}, g\right)$. By [7, $\tilde{M}=\mathbb{C}^{k} \times \tilde{N}$ and $\tilde{g}=$ $g_{e} \times \tilde{h}$, where $g_{e}$ is the standard metric and $(\tilde{N}, \tilde{h})$ is a steady gradient KählerRicci soliton with bounded nonnegative holomorphic bisectional curvature and with positive Ricci curvature. Hence there exists some smooth real-valued function $f$ satisfying (4.1) on $\tilde{N}$. Now let us also assume that the scalar curvature of $M$ attains its maximum at some point. Hence the same is true for $\tilde{N}$. Then by $\underline{8}, \nabla f($ the real gradient of $f$ relative to $\tilde{h}$ ) has a unique zero at some $\tilde{p}$. Let $\lambda_{1} \geq \lambda_{2} \geq \cdots \geq \lambda_{l}>0$ be the eigenvalues of the Ricci curvature at $\tilde{p}$, where $l=n-k$. Recall that $\tilde{N}$ is biholomorpic to $\mathbb{C}^{l}$ by [10, 2]. Let $\Gamma$ be the fundamental group of $M$. Then by the proof of Theorem 3.1, each $F$ in $\Gamma$ is of the form $\left(\gamma_{1}, \gamma_{2}\right)$, where $\gamma_{1}$ is a holomorphic isometry of $\mathbb{C}^{k}$ and $\gamma_{2}$ is a holomorphic isometry of $(\tilde{N}, \tilde{h})$. Let

$$
\Lambda_{1}=\left\{\gamma_{1} \mid\left(\gamma_{1}, \gamma_{2}\right) \in \Gamma \text { for some } \gamma_{2}\right\}
$$

and

$$
\Lambda_{2}=\left\{\gamma_{2} \mid\left(\gamma_{1}, \gamma_{2}\right) \in \Gamma \text { for some } \gamma_{1}\right\}
$$

We want to describe the holomorphic isometries in $\Lambda_{2}$. In fact, we want to describe any holomorphic isometry of $\tilde{N}$ in general. It was shown by Bryant [2] that there are global holomorphic coordinates $z^{i}$ of $\tilde{N}$, ranging over $\mathbb{C}^{l}$, which linearize the holomorphic vector field $Z=\frac{1}{2}(\nabla f-\sqrt{-1} J(\nabla f))$. That is to say,

$$
Z=\sum_{i=1}^{l} \lambda_{i} \frac{\partial}{\partial z^{i}} .
$$

Such coordinates are called Poincaré coordinates.

Remark 4.1. We remark that in the case of an expanding gradient Kähler-Ricci soliton with nonnegative Ricci curvature, Poincaré coordinates also exist. This is because, in this case, the potential function $f$ has a unique critical point and $J(\nabla f)$ is also a Killing vector field.

Now let $\phi:(\tilde{N}, \tilde{h}) \rightarrow(\tilde{N}, \tilde{h})$ be a holomorphic isometry. Thus $\phi^{*}(\tilde{h})=\tilde{h}$.

Lemma 4.1. The following are true:

(1) $\phi^{*}(f)=f$.

(2) $\phi_{*}(Z)=Z$.

Proof. Let $f_{1}=\phi^{*}(f)$. Since $\phi^{*}(\tilde{h})=\tilde{h}$ we have $\nabla^{2}\left(f_{1}\right)=\phi^{*}\left(\nabla^{2} f\right)$ and thus $\left(f_{1}\right)_{i \bar{\jmath}}=R_{i \bar{\jmath}}=f_{i \bar{\jmath}}$. Hence $\nabla f_{1}$ has a unique zero, which must be at $\tilde{p}$. Hence $\phi(\tilde{p})=\tilde{p}$. So $f_{1}(\tilde{p})=f(\tilde{p})$. On the other hand, since $\nabla f(\tilde{p})=\nabla f_{1}(\tilde{p})=0$ and $\nabla^{2}\left(f-f_{1}\right)=0$, we conclude that (1) is true. (2) follows from the definition of $Z$, the fact that $\phi^{*}(\tilde{h})=\tilde{h}$ and from (1).

Lemma 4.2. Let $\phi$ be a holomorphic isometry of $(\tilde{N}, \tilde{h})$. Let $\phi(z)=w$ in the Poincaré coordinates. Then $\phi$ is an upper triangular polynomial map in the sense that

$$
w=A(z)+G(z),
$$


where $A(z)$ is a linear map and $G=\left(G_{1}, \ldots, G_{l}\right)$ with $G_{i}=G_{i}\left(z^{i+1}, \ldots, z^{l}\right)$. If, in addition, for all $j$,

$$
\sum_{i=1}^{l} m_{i} \lambda_{i} \neq \lambda_{j}
$$

for all nonnegative integers $m_{i}$ such that $\sum_{i=1}^{l} m_{i} \geq 2$, then $\phi$ is a linear map.

Proof. This is basically a result in [2]. For the sake of completeness, we sketch the proof here. Since $\phi_{*}(Z)=Z$ and we are using Poincaré coordinates in the domain and target, for each $i$, we have

$$
\lambda_{i} w^{i}=\sum_{j=1}^{l} \lambda_{j} z^{j} \frac{d w^{i}}{d z^{j}}
$$

everywhere. On the other hand, for each $i$, as a function of $z$ we know that $w^{i}$ has a global power series representation

$$
w^{i}=\sum_{|\alpha|=1}^{|\alpha|=\infty} c_{\alpha}(i) z^{\alpha},
$$

where $\alpha$ is a multi-index. The fact that there is no constant term in (4.3) follows from the fact that, by their definition, both $z$ and $w$ must vanish at $\tilde{p} \in \tilde{N}$. Now by substituting the power series in (4.3) and its derivative with respect to $z$ for both sides of (4.2), equating coefficients and using the fact that the $\lambda_{i}$ 's are all positive, the result follows.

In [32, Yang proves the following: Let $M$ be a noncompact Kähler-Ricci soliton with nonnegative Ricci curvature. Suppose its scalar curvature attains a positive maximum at a compact complex submanifold $K$ with codimension 1 and the Ricci curvature is positive away from $K$. Then $M$ is a holomorphic line bundle over $K$.

In case the holomorphic bisectional curvature is bounded and nonnegative, from the lemma, the proof of Theorem 3.1 and the discussion before Lemma 4.1, we have the following:

Theorem 4.1. Let $(M, g)$ be a steady gradient Kähler-Ricci soliton with bounded nonnegative holomorphic bisectional curvature such that the scalar curvature is maximal at some $p \in M$. Then assuming the above notation, there exist global complex coordinates on $\tilde{N}$ such that $M$ is a Riemannian and holomorphic fiber bundle over $\mathbb{C}^{k} / \Lambda_{1}$ with fiber $\mathbb{C}^{n-k}$ such that each $\gamma_{2} \in \Lambda_{2}$ is of upper triangular form. If, in addition, for all positve $\lambda_{j}$ we have

$$
\sum_{i=1}^{l} m_{i} \lambda_{i} \neq \lambda_{j}
$$

for all nonnegative integers $m_{i}$ such that $\sum_{i=1}^{l} m_{i} \geq 2$, then $M$ is a holomorphic vector bundle over $\mathbb{C}^{k} / \Lambda_{1}$.

We end this section by proving a general volume growth estimate for expanding gradient solitons. The motivation is from the work of $\mathrm{Ni}$ [24], where he proved that a complete noncompact Kähler manifold with bounded and nonnegative holomorphic bisectional curvature and with maximum volume growth must have quadratic curvature decay as in (1) in the introduction. 
Proposition 4.1. Suppose $\left(M^{n}, g\right)$ is a complete Riemannian manifold such that $g$ is an expanding gradient Ricci soliton with nonnegative scalar curvature. Then for any $o \in M$, there is a constant $C>0$ such that $V_{o}(r) \geq C r^{n}$ for all $r \geq 1$, where $V_{o}(r)$ is the volume of a geodesic ball of radius $r$ with center at $o$.

Proof. Let $f$ be such that $f_{i j}=R_{i j}+g_{i j}$. By the proof in [23, §20] (see also [19]), $\mathcal{R}+|\nabla f|^{2}-2 f$ is a constant, where $R$ is the scalar curvature. Modifying $f$ by adding a constant to $f$, we may assume that $\mathcal{R}+|\nabla f|^{2}-2 f=0$. Since $\mathcal{R} \geq 0$, we have $f \geq 0$ and $|\nabla f|^{2} \leq 2 f$. Fix $o \in M$. For any $x$ let $\gamma(s)$ be a minimal geodesic from $o$ to $x$ parametrized by arc length and let $r=d(o, x)$. Then for any $0 \leq \tau \leq r$,

$$
\begin{aligned}
\frac{d}{d \tau} f(\gamma(\tau)) & \leq|\nabla f| \\
& \leq(2 f)^{\frac{1}{2}} .
\end{aligned}
$$

Hence $f^{\frac{1}{2}}(x) \leq \frac{1}{\sqrt{2}} r+f^{\frac{1}{2}}(o)$. This implies that $|\nabla f|(x) \leq r+(2 f(o))^{\frac{1}{2}}$.

On the other hand, $\Delta f \geq n$, where $n$ is the dimension of $M$. For any $R$,

$$
\begin{aligned}
n V(R) & \leq \int_{B(R)} \Delta f \\
& =\int_{\partial B(R)} \frac{\partial f}{\partial \nu} \\
& \leq \int_{\partial B(R)}|\nabla f| \\
& \leq\left(R+(2 f(o))^{\frac{1}{2}}\right) A(R),
\end{aligned}
$$

where $A(R)$ is the area of $\partial B_{o}(R)$ and $V(R)=V_{o}(R)$. Hence

$$
V(R) \geq\left(\frac{R+(2 f(o))^{\frac{1}{2}}}{1+(2 f(o))^{\frac{1}{2}}}\right)^{n} V(1)
$$

for $R \geq 1$.

\section{REFERENCES}

1. Brown, M., The monotone union of open $n$-cells is an open $n$-cell, Proc. Amer. Math. Soc. 12 (1961), 812-814. MR0126835 (23:A4129)

2. Bryant, R., Gradient Kähler Ricci solitons, Astérisque No. 321 (2008), 51-97. MR 2521644

3. Carrillo, J.A. and Ni, L., Sharp logarithmic Sobolev inequalities on gradient solitons and applications, preprint.

4. Cao, H.-D., On Harnack's inequality for the Kähler-Ricci flow, Invent. Math. 109 (1992), 247-263. MR:1172691 (93f:58227)

5. Cao, H.-D., Existence of gradient Kähler-Ricci solitons, Elliptic and parabolic methods in geometry (Minneapolis, MN, 1994), 1-16. MR1417944 (98a:53058)

6. Cao, H.-D., Limits of solutions to the Kähler-Ricci flow, J. Differential Geom. 45 (1997), 257-272. MR1449972 (99g:53042)

7. Cao, H.-D., On dimension reduction in the Kähler-Ricci flow, Comm. Anal. Geom. 12 (2004), 305-320. MR2074880 (2005d:53104)

8. Cao, H.-D. and Hamilton, R. S., Gradient Kähler-Ricci solitons and periodic orbits, Comm. Anal. Geom. 8 (2000), no. 3, 517-529. MR.1775136 (2001g:53117)

9. Chau, Albert and Tam, Luen-fai, Gradient Kähler-Ricci solitons and a uniformization conjecture, arXiv eprint 2003. arXi:math.DG/0310198.

10. Chau, A. and Tam, L.-F., A note on the uniformization of gradient Kähler-Ricci solitons, Math. Res. Lett. 12 (2005), no. 1, 19-21. MR2122726 (2005i:53081) 
11. Chau, A. and Tam, L.-F., On the complex structure of Kähler manifolds with non-negative curvature, J. Differential Geom. 73 (2006), 491-530. MR2228320 (2007e:53085)

12. Chau, A. and Tam, L.-F.,Non-negatively curved Kähler manifolds with average quadratic curvature decay, Comm. Anal. Geom. 15 (2007), no. 1, 121-146. MR.2301250 (2007m:53086)

13. Chau, A. and Tam, L.-F., On the Steinness of a class of Kähler manifolds, J. Differential Geom. 79 (2008), 167-183. MR2420016 (2009g:32054)

14. Chau, A. and Tam, L.F., Survey on the Kähler Ricci flow and Yau's uniformization conjecture, Surveys in Differential Geometry vol. 12, Int. Press, Somerville, MA, 2008. MR2488949 (2009k:53164)

15. Cheeger, J. and Gromoll, D., The splitting theorem for manifolds of nonnegative Ricci curvature, J. Differential Geometry 6 (1971/72), 119-128. MR0303460 (46:2597)

16. Cheeger, J. and Gromoll, D., On the structure of complete manifolds of nonnegative curvature, Ann. of Math. (2), 46 (1972), 413-433. MR0309010 (46:8121)

17. Cheeger, J., Gromov, M. and Taylor, M., Finite propagation speed, kernel estimates for functions of the Laplace operator, and the geometry of complete Riemannian manifolds, J. Differential Geom. 17 (1982), 15-53. MR658471 (84b:58109)

18. Chen, B.L. and Zhu, X.P., Uniqueness of the Ricci flow on complete noncompact manifolds, J. Differential Geom. 74 (2006), 119-154. MR2260930 (2007i:53071)

19. Chow, B., Chu, S.-C., Glickenstein, D., Guenther, C., Isenberg, J., Ivey, T., Knopf, D., Lu, P., Luo, F. and Ni, L., The Ricci flow: techniques and applications. Part I. Geometric aspects, Mathematical Surveys and Monographs, 135, American Mathematical Society, Providence, RI, 2007. MR2302600 (2008f:53088)

20. Chow, B., Lu, P., and Ni, L., Hamilton's Ricci flow, Graduate Studies in Mathematics, 77, American Mathematical Society, Providence, RI; Science Press, New York, 2006. MR 2274812 (2008a:53068)

21. Fan, X.-Q., A uniqueness result of Kähler Ricci flow with an application, Proc. Amer. Math. Soc. 135 (2007), no. 1, 289-298 (electronic). MR2280196 (2007h:53099)

22. Hamilton, R. S., A compactness property for solutions of the Ricci flow, Amer. J. Math. 117 (1995), 545-572. MR1333936 (96c:53056)

23. Hamilton, R. S., Formation of Singularities in the Ricci Flow, Surveys in Differential Geometry, Vol. II (1995), 7-136. MR1375255 (97e:53075)

24. Ni, L., Ancient solutions to Kähler-Ricci flow, Math. Res. Lett. 12 (2005), 633-653. MR2189227 (2006i:53097)

25. Ni, L. and Tam, L.-F., Plurisubharmonic functions and the structure of complete Kähler manifolds with nonnegative curvature, J. Differential Geom. 64 (2003), 457-524. MR2032112 (2005a:32023)

26. Shi, W.-X., Ricci deformation of the metric on complete noncompact Riemannian manifolds, J. Differential Geometry 30 (1989), 223-301. MR.1001277 (90i:58202)

27. Shi, W.-X., Ricci deformation of the metric on complete noncompact Kähler manifolds, Ph.D. thesis, Harvard University, 1990.

28. Shi, W.-X., Ricci Flow and the uniformization on complete noncompact Kähler manifolds, J. Differential Geometry 45 (1997), 94-220. MR1443333 (98d:53099)

29. Tian, G. and Yau, S.-T., Complete Kähler manifolds with zero Ricci curvature. I., J. Amer. Math. Soc. 3 (1990), 579-609. MR1040196 (91a:53096)

30. Takayama, S., The Levi problem and the structure theorem for non-negatively curved complete Kähler manifolds, J. Reine Angew. Math. 504 (1998), 139-157. MR.1656759 (99j:32034)

31. Wu, H. and Zheng, F.-Y., Examples of positively curved complete Kähler manifolds, preprint.

32. Yang, Bo, A characterization of Koiso's typed solitons, preprint, arXiv:0802.0300.

33. Yau, S.-T., A review of complex differential geometry, Proc. Sympos. Pure Math., 52 Part 2 (1991), 619-625. MR:1128577 (92j:32029)

Department of Mathematics, The University of British Columbia, Room 121, 1984 Mathematics Road, Vancouver, British Columbia, Canada V6T $1 Z 2$

E-mail address: chau@math.ubc.ca

The Institute of Mathematical Sciences and Department of Mathematics, The Chinese University of Hong Kong, Shatin, Hong Kong, China

E-mail address: lftam@math.cuhk.edu.hk 